\title{
Prevalence of Sleep Disturbances in Mild Cognitive Impairment and Dementing Disorders: A Multicenter Italian Clinical Cross-Sectional Study on 431 Patients
}

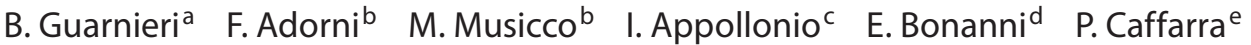 \\ C. Caltagirone $^{f} \quad$ G. Cerroni ${ }^{a} \quad$ L. Concarie F.I.I. Cosentino $^{g} \quad$ S. Ferrara $^{n} \quad$ S. Fermi $^{c} \quad$ R. Ferri $^{g}$ \\ G. Gelosac $^{c} \quad$ G. Lombardi ${ }^{\text {h }} \quad$ D. Mazzei ${ }^{i} \quad$ S. Mearelli ${ }^{j} \quad$ E. Morrone ${ }^{k} \quad$ L. Murri ${ }^{d} \quad$ F.M. Nobili ${ }^{i}$ \\ S. Passerol R. Perri ${ }^{m} \quad$ R. Rocchil P.Sucapane ${ }^{j} \quad$ G. Tognoni ${ }^{d} \quad$ S. Zabberoni ${ }^{m} \quad$ S. Sorbi $^{\text {h }}$ \\ ${ }^{a}$ Center of Sleep Medicine, Villa Serena Hospital, Città S. Angelo, b Italian National Research Center (ITB-CNR), and \\ 'Neuroscience Department, S. Gerardo Hospital Monza, Bicocca University, Milan, d Neuroscience Department, \\ University of Pisa, Pisa, ${ }^{e}$ Neuroscience Department, University of Parma, Parma, ${ }^{f}$ Tor Vergata University, S. Lucia \\ Foundation, IRCCS, Rome, ${ }^{9}$ Department of Neurology, Oasi Institute IRCCS, Troina, h Department of Neurological \\ and Psychiatric Sciences, University of Florence, Florence, 'Clinical Neurophysiology, Department of Neuroscience, \\ Ophthalmology and Genetics, University of Genoa, Genoa, 'Department of Neurology, University of L'Aquila, \\ L'Aquila, 'kSleep Physiopathology, S. Martino Hospital, Genoa, 'Neuroscience Department, University of Siena, Siena, \\ ${ }^{\mathrm{m}} \mathrm{S}$. Lucia Foundation, IRCCS, Rome, and ${ }^{\mathrm{n}}$ Department of Neurology, Villa Serena Hospital, Città S. Angelo, Italy
}

\section{Key Words}

Sleep disorders - Excessive daytime sleepiness • Mild cognitive impairment • Alzheimer's disease •

Frontotemporal dementia • Lewy body dementia •

Parkinson's disease dementia • Vascular dementia

\begin{abstract}
Background/Aims: Sleep disturbances are common in the elderly and in persons with cognitive decline. The aim of this study was to describe frequency and characteristics of insomnia, excessive daytime sleepiness, sleep-disordered breathing, REM behavior disorder and restless legs syndrome in a large cohort of persons with mild cognitive impairment or dementia. Methods: 431 consecutive patients were enrolled in 10 Italian neurological centers: 204 had Alzheimer's disease, 138 mild cognitive impairment, 43 vas-
\end{abstract}

cular dementia, 25 frontotemporal dementia and 21 Lewy body dementia or Parkinson's disease dementia. Sleep disorders were investigated with a battery of standardized questions and questionnaires. Results: Over $60 \%$ of persons had one or more sleep disturbances almost invariably associated one to another without any evident and specific pattern of co-occurrence. Persons with Alzheimer's disease and those with mild cognitive impairment had the same frequency of any sleep disorder. Sleep-disordered breathing was more frequent in vascular dementia. REM behavior disorder was more represented in Lewy body or Parkinson's disease dementia. Conclusion: A careful clinical evaluation of sleep disorders should be performed routinely in the clinical setting of persons with cognitive decline. Instrumental supports should be used only in selected patients.

Copyright $\odot 2012$ S. Karger AG, Basel

\section{KARGER}

Fax +4161306 1234

E-Mail karger@karger.ch

www.karger.com (c) 2012 S. Karger AG, Basel

$1420-8008 / 12 / 0331-0050 \$ 38.00 / 0$

Accessible online at:

www.karger.com/dem
Dr. Biancamaria Guarnieri

Center of Sleep Medicine, Villa Serena Hospital

Viale L. Petruzzi 42

IT-65013 Città S. Angelo (Italy)

Tel. +39085959 0237, E-Mail bmariaguarnieri@villaserena.it 


\section{Introduction}

The prevalence of sleep disturbances increases with age suggesting that sleep is affected by aging itself and/or by aging-related conditions $[1,2]$. Excessive daytime sleepiness (EDS) is highly frequent in neurodegenerative disorders $[3,4]$ that are the most common chronic brain diseases of elderly people. In particular, sleep disturbances have been reported in $25-44 \%$ of patients with Alzheimer's disease (AD) [5].

The high prevalence of sleep disorders reported in neurodegenerative diseases could be explained by intrinsic changes specific to each form of dementia but also by the high prevalence of non-specific conditions potentially affecting sleep in these patients (depression, chronic pain and bedrest, medication use) [6]. In addition, sleep can be affected by circadian rhythm changes and primary sleep disorders (PSD): in elderly persons, PSD are mainly represented by sleep-disordered breathing (SDB), REM parasomnias, in particular REM behavior disorder (RBD), restless legs syndrome (RLS) and periodic limb movements (PLM) [7]. It is highly plausible that the diffuse brain damage that characterizes dementias might extend over the cognitive brain areas, to involve the neural networks that control sleep function (anterior hypothalamus, reticular activating system, suprachiasmatic nucleus and pineal gland). Sleep disorders seem to be different in each form of dementia and this might be the consequence of the different brain pathological involvement that characterizes each dementia type [8-12].

Our understanding of the basic mechanisms of sleep/ wake disturbances and their epidemiology in cognitive decline is quite important in the evaluation of persons with dementia since their presence might contribute to elucidate the type of structural neurological alterations. As an example, SDB is expected to be more frequent in diseases involving respiratory and autonomic neurons as Lewy body dementia (LBD) Parkinson's disease dementia (PDD) and multiple system atrophy (MSA), but they are also present in AD. RBD is frequently reported in Parkinson's disease (PD), LBD, MSA [13] and several studies showed that this disorder may even precede the clinical onset of synucleinopathies [14].

Sleep disorders are not exclusively seen in neurodegenerative dementias since a high prevalence of obstructive sleep apnea (OSA) is observed also in patients with vascular cognitive impairment $[15,16]$.

Mild cognitive impairment (MCI) is considered a risk condition for dementia, especially $\mathrm{AD}$ [17]; RBD and other sleep disorders are associated with cognitive impair- ment and, in persons with MCI, the presence of particular sleep disturbances might identify persons at higher risk of evolving to $\mathrm{AD}$ or other forms of dementia [18].

Although there is wide agreement that sleep disorders represent a relevant problem in neurodegenerative and vascular dementias, no previous studies investigated these disorders simultaneously in a wide population of persons with the main forms of dementia and in MCI, using standardized instruments. The aim of this multicenter study was to describe frequency and characteristics of sleep disorders in a large cohort of communitydwelling persons with dementia or MCI.

\section{Subjects and Methods}

\section{Participants}

Consecutive community-dwelling patients with dementia or MCI were enrolled by 10 Italian neurological centers over a period of 6 months. The diagnosis of dementia was made according to the DSM-IV-TR criteria [19]. AD and VaD were diagnosed respectively according to NINCS-ARDRA and NINDS-AIREN criteria [20, 21]. LBD and PDD, frontotemporal dementia (FTD) and MCI were diagnosed according to specific consensus guidelines [22, 23].

Each participating center declared in advance the total number of patients that was possible to enroll. This predefined total number was divided in each center as follows: $50 \% \mathrm{AD}, 25 \% \mathrm{MCI}$, $15 \%$ FTD, LBD/PDD, 10\% VaD. Patients with life expectancy of less than 6 months, with a score of dementia severity at the Clinical Dementia Rating Scale [24] of more than 3 and patients not supported by a reliable caregiver were excluded. All the patients were evaluated in each participating center by a single neurologist expert in dementia and sleep disturbances.

Because the aim of the study is to describe type, frequency and characteristics of sleep disorders in patients with different type of dementia and MCI, no control (i.e. not cognitively impaired) group of patients was enrolled.

\section{Demographic and Clinical Data}

For each included patient we registered age, sex, education and some basic clinical information regarding the characteristics of dementia. In particular, for what concerns the variables considered in this study, we estimated the severity of dementia with the Mini-Mental State Examination (MMSE) [25], and the severity of depressive symptoms with the Beck Depression Inventory-II (BD-III) [26]. Functional impairment was evaluated with the Physical Self-Maintenance Scale (PSMS) [27] and in a selected group of patients, the Italian version of the Disability Assessment for Dementia scale has been used [28].

\section{Sleep Disorders Evaluation}

Sleep was investigated directly to the patient and/or the direct and reliable caregiver with a battery of questionnaires whenever possible standardized and validated with definite and acceptable values of sensitivity and specificity.

Insomnia has been investigated and diagnosed according to an instrument validated in a wide Italian population [29]. For the 
screening of SDB, we used the Berlin questionnaire which showed a sensitivity of $86 \%$ and a specificity of $77 \%$ in a western population [30]. A condition of clinically probable SDB was diagnosed in persons having a positive history of usual snoring or sleep apneas, who met the criteria for high risk of sleep apnea according to the Berlin questionnaire [30]. In brief, the questionnaire addresses the presence and frequency of snoring behavior, daytime sleepiness or fatigue and history of obesity and/or hypertension. Patients with persistent and frequent symptoms in two of these three domains were considered at high risk for SDB.

The presence of RLS was initially investigated with a simple question: 'In the evening, sitting or lying in bed, have you ever had a tidy sensation of urgency to move your legs to have relief and does this uncomfortable situation cause difficulties in starting or maintaining sleep?' In patients answering positively to this question, a clinically definite RLS (CD-RLS) was diagnosed in patients responding positively to four questions representing the criteria for the clinical diagnosis of RLS according to the International RLS Study Group [31, 32].

At the time the study was carried out there were no available validated instruments or consensus on the criteria for the clinical diagnosis of RBD in cognitively impaired persons. In our study, a clinically probable RBD was diagnosed in patients presenting during sleep, about $1 \mathrm{~h}$ after falling asleep, rude movements and/ or vocalizations as if they were enacting terrifying nightmares, sometimes provoking harm to self or to their bed partner: these episodes do not necessarily cause the complete awakening of the subject.

The validity of this approach in assessing the presence of RBD in an aging and dementia cohort has been recently evaluated in a formal study that has reported a sensitivity of $98 \%$ and a specificity of $74 \%$, considering polysomnography as the gold standard [33].

Since specific questionnaires to assess EDS have not been formally validated in persons with cognitive decline, EDS was diagnosed from a direct clinical observation of the patient and/or on the caregiver's information about naps during the day.

In patients with any of the above-described sleep disturbances, we investigated the sleep quality with the Pittsburgh Sleep Quality Index (PSQI). With this questionnaire a cut-off score of 5 has a sensitivity of $89.6 \%$ and a specificity of $86.5 \%$ in distinguishing good and poor sleepers in noncognitively impaired persons $[34,35]$. PSQI has been widely used also in old and cognitively impaired persons even though it has not been formally validated in such populations.

\section{Data Analysis}

Comparisons of numerical continuous variables were carried out with the analysis of variance with Bonferroni adjustment for multiple comparisons. Each included patient was classified according to the presence of the different investigated sleep disorders. The frequency of these disorders was compared in the five different diagnostic categories. The strength of association between any sleep disorder and type of dementia was estimated as relative risk and calculated as odds ratios, considering as reference category patients with AD. Relative risk estimates were calculated with logistic regression analysis adjusting for age, sex, severity of cognitive, functional impairment and of depressive symptoms. Ninety-five percent confidence limits of odds ratios were calculated using the standard errors of the coefficients of the logistic regression.
Table 1. Age and gender characteristics by sleep disturbance

\begin{tabular}{llll}
\hline & $\begin{array}{l}\text { Women } \\
n(\%)\end{array}$ & $\begin{array}{l}\text { Age } \\
\text { years }\end{array}$ & Total \\
& & & \\
\hline SDB & & & \\
$\quad$ Yes & $130(50.8)^{*}$ & $76.0 \pm 8.4$ & $255[60.0]$ \\
$\quad$ No & $111(63.4)$ & $75.9 \pm 9.8$ & \\
EDS & $106(49.8)^{*}$ & $76.4 \pm 8.7$ & $213[50.1]$ \\
$\quad$ Yes & $132(62.6)$ & $75.6 \pm 9.3$ & \\
$\quad$ No & $119(56.1)$ & $77.0 \pm 6.9^{*}$ & $212[49.9]$ \\
Insomnia & $120(56.3)$ & $74.8 \pm 10.6$ & \\
$\quad$ Yes & & & \\
No & $44(45.8)^{*}$ & $75.2 \pm 7.4$ & $96[22.6]$ \\
RBD & $193(59.0)$ & $76.2 \pm 9.4$ & \\
$\quad$ Yes & $16(61.5)^{*}$ & $74.9 \pm 8.1$ & $26[6.1]$ \\
No & $223(55.9)$ & $76.0 \pm 9.1$ & \\
RLS & Yes & &
\end{tabular}

$\mathrm{SDB}=$ Sleep-disordered breathing; $\mathrm{RBD}=\mathrm{REM}$ behavior disorder; RLS = restless legs syndrome; EDS = excessive daytime sleepiness. Numbers in round parentheses are row percentages. Numbers in squared parentheses are column percentages, and \pm are standard deviations.

${ }^{*} \mathrm{p}<0.05$.

\section{Results}

We enrolled 431 patients: 204 (47.3\%) had AD, 138 (32.0\%) MCI, 43 (10.0\%) VaD, 25 (5.8\%) and 21 (4.9\%) had FTD or LBD/PDD, respectively. The sex ratio was about 1:1 in MCI patients, but there were slightly more men in the FTD and LBD/PDD groups. Men prevailed on women in the group with $\mathrm{VaD}$, whereas women were the large majority among AD patients. These differences in sex distribution among different diagnostic categories were all statistically significant. In particular, women were significantly more represented in $\mathrm{AD}$ with respect to all the other diagnostic categories.

Mean age was over 70 in all the groups with the youngest mean age (71 years) for FTD and the oldest for LBD/ PDD (78.8 years) patients (fig. 1). Persons with MCI were significantly younger than those with $\mathrm{AD}$ : patients with FTD were significantly younger than patients with other types of dementia. Mean education varied from a minimum of 5.8 years of formal schooling in patients with $\mathrm{VaD}$ to a maximum of 8.2 years in patients with $\mathrm{MCI}$ and the difference between $\mathrm{VaD}$ and $\mathrm{MCI}$ was statistically significant $(p=0.021)$. In persons with dementia, the lowest 

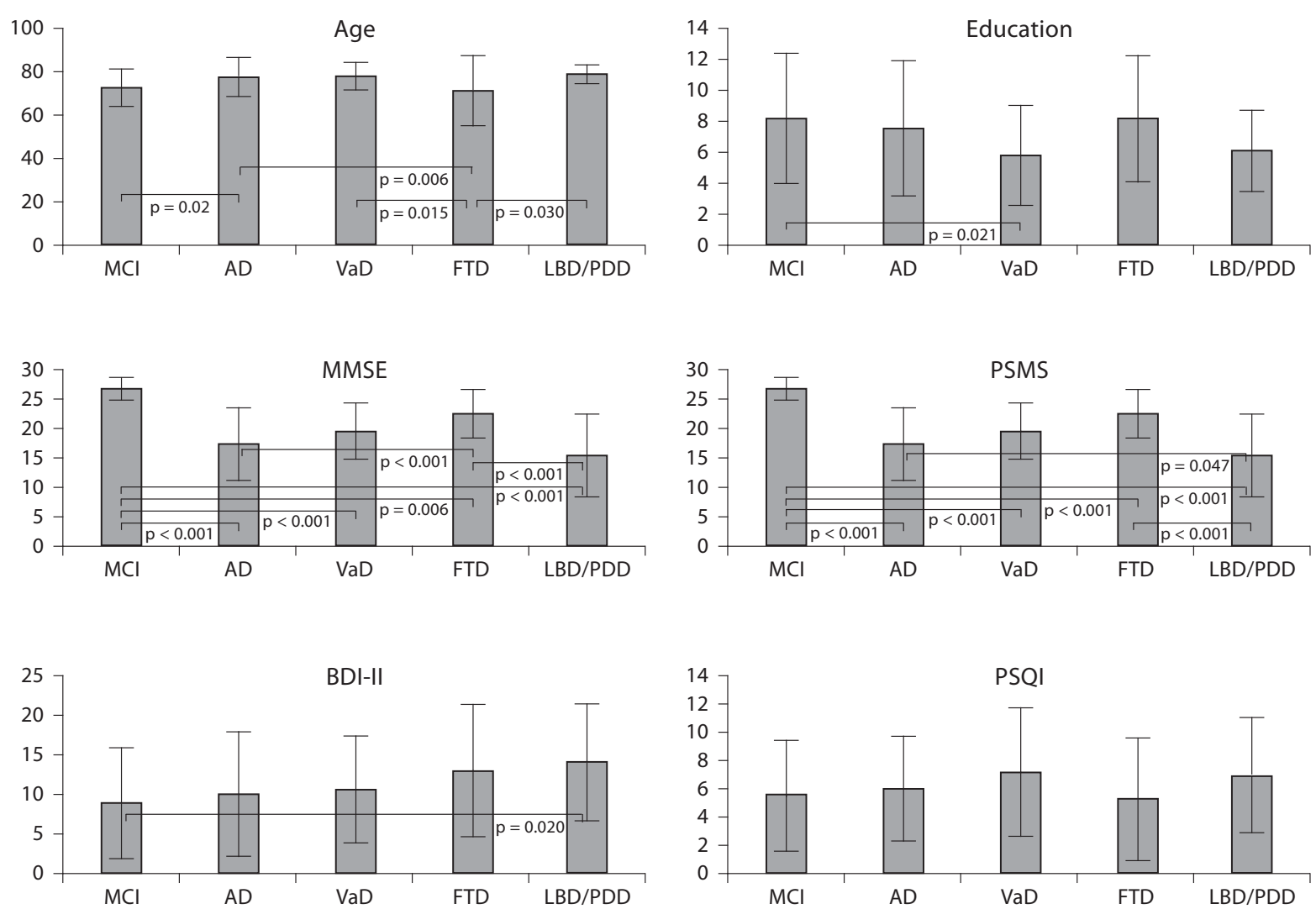

Fig. 1. Mean age (years), education (years) and mean scores at MMSE, PSMS, BDI-II and PSQI by MCI and different types of dementia. Vertical lines represent standard deviations. MMSE = Mini-Mental State Examination; PSMS = Physical Self-Maintenance Scale; BDI-II = Beck Depression Inventory-II; PSQI = Pittsburgh Sleep Quality Index; $\mathrm{MCI}=$ mild cognitive impairment; $\mathrm{AD}=$ Alzheimer's disease; $\mathrm{VaD}=$ vascular dementia; FTD = frontotemporal dementia; LBD = Lewy Body dementia; PDD = Parkinson's disease dementia.

mean scores at MMSE were for patients with $\mathrm{LBD} / \mathrm{PDD}$, the highest for FTD. Persons with MCI had a mean MMSE score over the normality cut-off value of 23, and the score was significantly higher than that of all the other diagnostic groups. Patients with FTD had MMSE scores significantly higher than those with $\mathrm{AD}(\mathrm{p}<0.001)$ and LBD/PDD ( $<<0.001)$. Functional capacities measured with the PSMS were only slightly compromised in MCI patients and significantly less compromised than in patients with dementia. AD and FTD patients were significantly less functionally compromised than LBD/PDD patients $(\mathrm{p}=0.047$ and $\mathrm{p}<0.001$, respectively). Depressive symptoms were less severe in MCI patients followed by $\mathrm{AD}, \mathrm{VaD}, \mathrm{FTD}$ and $\mathrm{LBD} / \mathrm{PDD}$ patients, but the only significant difference was between $\mathrm{MCI}$ and $\mathrm{LBD} / \mathrm{PDD}$ $(p=0.020)$. Although there were different scores at the PSQI according to the different diagnoses, these differences were nonstatistically significant.

The most frequent sleep disorder was SDB which was present in almost $60 \%$ of the patients, followed by EDS (50.1\%), insomnia (49.9\%), RBD (22.6\%) and RLS (6.1\%) (table 1). Men more frequently had SDB, RBD and EDS, whereas RLS was more frequent in women. No difference was observed in the percentage of men and women with or without insomnia. No difference in the mean age was observed between persons with and without sleep disorders with the only exception of persons with insomnia who were significantly older than those unaffected (77 \pm 6.9 vs. $74.8 \pm 10.6$ years, $\mathrm{p}<0.05)$. 


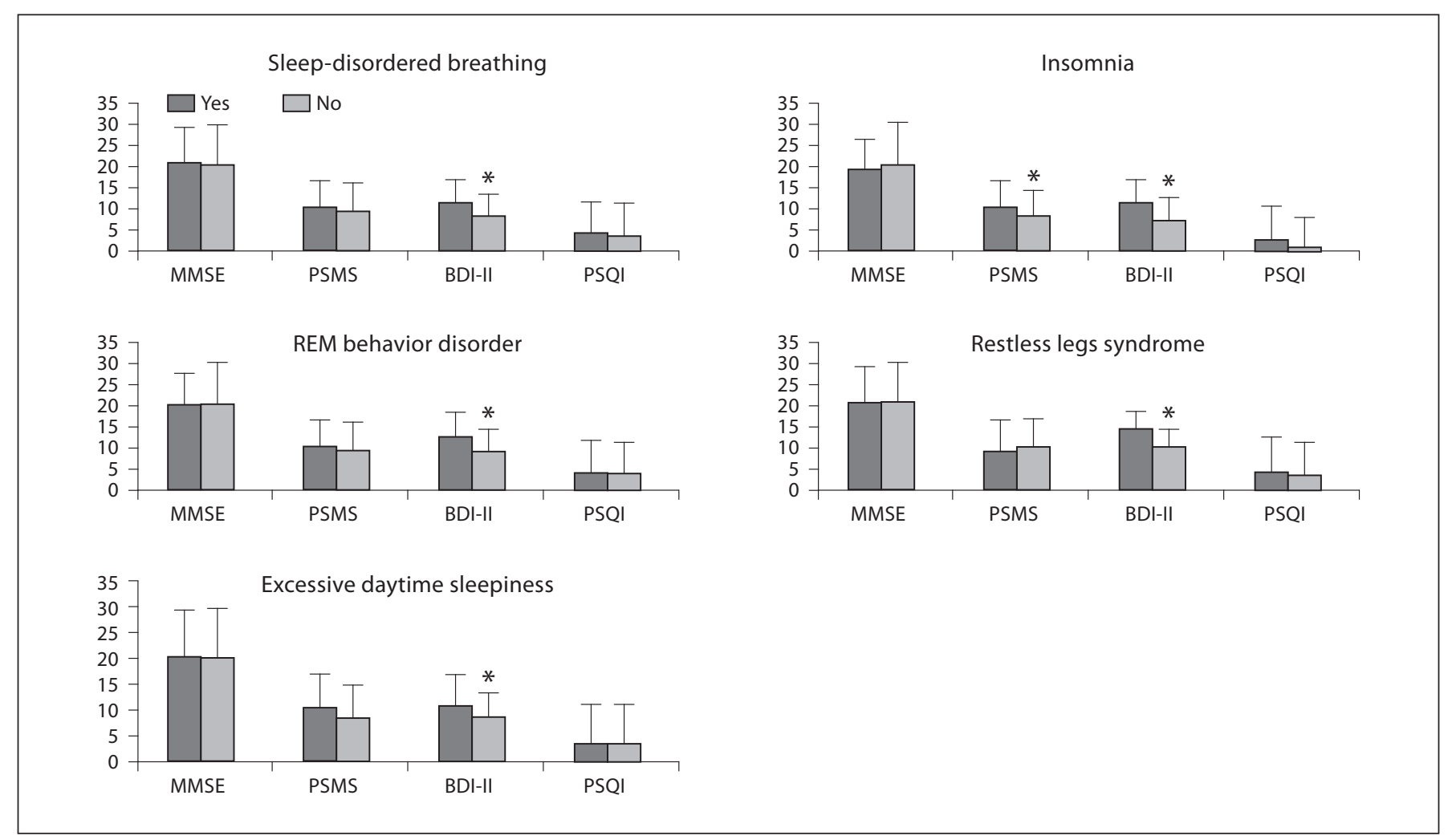

Fig. 2. Severity of depressive symptoms (BDI-II), of cognitive (MMSE) and functional (PSMS) impairment and sleep quality (PSQI) by sleep disturbance. MMSE = Mini-Mental State Examination; PSMS = Physical SelfMaintenance Scale; BDI-II = Beck Depression Inventory-II; PSQI = Pittsburgh Sleep Quality Index. Vertical lines represent standard deviations. ${ }^{*} \mathrm{p}<0.05$.

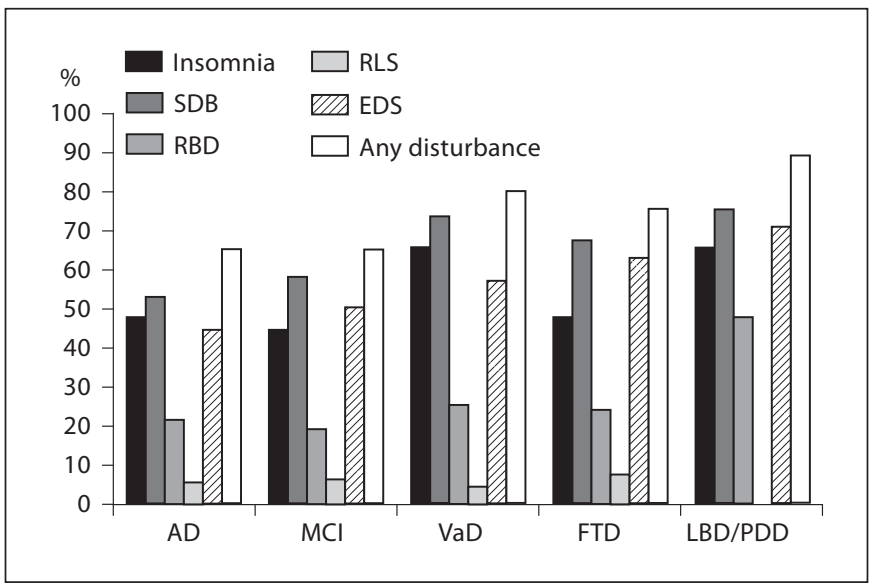

Fig. 3. Prevalence of insomnia and other sleep disturbances by type of dementia. $\mathrm{MCI}=$ Mild cognitive impairment; $\mathrm{AD}=\mathrm{Alz}$ heimer's disease; $\mathrm{VaD}=$ vascular dementia; FTD = frontotemporal dementia; $\mathrm{LBD}=$ Lewy Body dementia; $\mathrm{PDD}=$ Parkinson's disease dementia; $\mathrm{SDB}=$ sleep-disordered breathing; $\mathrm{RBD}=\mathrm{REM}$ behavior disorder; RLS = restless legs syndrome; EDS = excessive daytime sleepiness.
Depressive symptoms measured with the BDI-II were significantly more severe in persons with any type of sleep disturbance (fig. 2): persons with insomnia showed significantly more severe functional impairment at the PSMS.

No other significant difference was observed in the level of cognitive and functional impairment, or in the quality of sleep (PSQI).

The different sleep disturbances occurred almost always in association (table 2): the two disturbances that were more frequently found isolated were insomnia and EDS, 10 and $8 \%$ of the cases, respectively. RLS was always associated to other disturbances and all the persons with this syndrome had also SDB. Although occurring with high frequency associated one to another, no evident specific pattern of association emerged.

Every type of sleep disorder was present in each type of dementia and in persons with MCI, with the exception of RLS that was not recognized in LBD/PDD patients (fig. 3). Taking $\mathrm{AD}$ patients as the reference category (ta- 
Table 2. Associations among different sleep disturbances

\begin{tabular}{lrlllrrr}
\hline & Insomnia & SDB & RBD & RLS & EDS & Isolated & Total \\
\hline Insomnia & & $190(89.6)$ & $68(32.1)$ & $22(10.4)$ & $140(66.4)$ & $22(10.4)$ & 212 \\
SDB & $190(74.5)$ & & $94(36.9)$ & $26(10.2)$ & $196(76.9)$ & $3(1.2)$ & 255 \\
RBD & $68(32.1)$ & $94(21.8)$ & & $19(19.8)$ & $70(72.8)$ & $2(2.1)$ & 96 \\
RLS & $22(84.6)$ & $26(100)$ & $19(73.1)$ & & $20(76.9)$ & 0 & 26 \\
EDS & $140(74.5)$ & $196(92.0)$ & $70(32.9)$ & $20(9.4)$ & & $17(8.0)$ & 213 \\
Total & $212(49.1)$ & $255(59.2)$ & $96(22.3)$ & $26(6.0)$ & $213(49.4)$ & $44(10.2)$ & 431 \\
\hline
\end{tabular}

Numbers in parentheses are row percentages. SDB = Sleep-disordered breathing; RBD = REM behavior disorder; RLS = restless legs syndrome; EDS = excessive daytime sleepiness.

Table 3. Prevalence and risk of sleep disorders for MCI, VaD, FTD, LBD/PDD relative to AD

\begin{tabular}{|c|c|c|c|c|c|}
\hline & \multicolumn{5}{|l|}{ Diagnosis } \\
\hline SDB & $\begin{array}{l}110(53.9) \\
1\end{array}$ & $\begin{array}{l}81(58.7) \\
{[1.1 ; 0.6-2.0]}\end{array}$ & $\begin{array}{l}32(74.4) \\
{\left[2.5^{*} ; 1.1-5.7\right]}\end{array}$ & $\begin{array}{l}17(68.0) \\
{[1.3 ; 0.5-3.3]}\end{array}$ & $\begin{array}{l}16(76.2) \\
{[1.7 ; 0.6-5.2]}\end{array}$ \\
\hline RLS & $\begin{array}{l}13(6.4) \\
1\end{array}$ & $\begin{array}{l}9(6.7) \\
{[1.2 ; 0.4-4.1]}\end{array}$ & $\begin{array}{l}2(4.8) \\
{[0.8 ; 0.2-3.7]}\end{array}$ & $\begin{array}{l}2(8.0) \\
{[1.0 ; 0.2-5.4]}\end{array}$ & $\begin{array}{l}0 \\
-\end{array}$ \\
\hline EDS & $\begin{array}{l}89(44.5) \\
1\end{array}$ & $\begin{array}{l}68(50.4) \\
{[1.4 ; 0.8-2.4]}\end{array}$ & $\begin{array}{l}25(58.1) \\
{[1.5 ; 0.8-3.1]}\end{array}$ & $\begin{array}{l}16(64.0) \\
{[2.0 ; 0.8-5.0]}\end{array}$ & $\begin{array}{l}15(71.4) \\
{[2.8 ; 0.9-8.1]}\end{array}$ \\
\hline
\end{tabular}

Numbers in round parentheses are percentage with the disturbance within the diagnostic group. Numbers in squared parentheses are relative risks estimated as odds ratios and 95\% CI. The estimates are adjusted for age, sex, MMSE and BDI-II score. MCI = Mild cognitive impairment; $\mathrm{AD}=$ Alzheimer's disease; $\mathrm{VaD}=$ vascular dementia; FTD = frontotemporal dementia;
LBD = Lewy body dementia; PDD = Parkinson's disease dementia; $\mathrm{SDB}=$ sleep-disordered breathing; RBD = REM behavior disorder; RLS = restless legs syndrome; EDS = excessive daytime sleepiness; MMSE = Mini-Mental State Examination; BDI-II = Beck Depression Inventory-II.

* Reference category. ble 3), and adjusting for gender, age, MMSE and BDI-II scores, the presence of at least one of any of the investigated disturbances was two times more common in patients with $\mathrm{VaD}$ or $\mathrm{LBD} / \mathrm{PDD}$, but this difference was nonstatistically significant. Patients with MCI had a frequency of sleep disturbances of any type equal to that of patients with $\mathrm{AD}$. Insomnia frequency was identical in $\mathrm{AD}$ and FTD patients but was about 2.5 and 1.5 times more frequent in $\mathrm{VaD}$ and $\mathrm{LBD} / \mathrm{PDD}$ patients, respec- tively. SDB was about two times more frequent in patients with $\mathrm{VaD}$ than in those with $\mathrm{AD}$. RBD was 2.6 times more frequent in LBD/PDD patients, whereas no difference of occurrence was observed for RLS among the different types of dementia. Excessive daytime sleepiness was more frequent among $\mathrm{VaD}$ and $\mathrm{LBD} / \mathrm{PDD}$ patients than among $\mathrm{AD}$ patients, but these differences were only of borderline statistical significance. 


\section{Discussion}

In this study, we investigated the occurrence of different sleep disorders in a consecutive series of patients with neurodegenerative dementias, vascular dementia or MCI. All the included patients were investigated with the same standardized instruments and procedures thus allowing a direct comparison of the frequency of occurrence of sleep disorders in the different types of cognitive impairments. We evaluated five sleep disturbances: insomnia, RBD, SDB, RLS and EDS plus global sleep quality by PSQI. In this population, almost $60 \%$ had symptoms clinically compatible with SDB and more than $50 \%$ had EDS and insomnia. The frequency of sleep disturbances found in this study is higher than that previously reported in AD. However, direct comparison among different studies is not possible due to the different sleep disturbances investigated and to the different modalities of investigation. In any case, to our knowledge, this is the only study that investigated a wide group of patients in a systematic way with several standardized questions and questionnaires, so a higher diagnostic sensitivity than in previous studies was expected.

RBD was particularly frequent and diagnosed in about $25 \%$ of the patients, whereas RLS, which was the less frequent disorder, had a prevalence of about $6 \%$ not dissimilar from that found in normal elderly people [32].

The score of depressive symptoms was more severe in persons presenting any of the investigated sleep disorders. Depressive symptoms are common in older adults, especially among persons who are cognitively impaired, and insomnia is 2.5 times more frequent in older adults with depressed mood [36]. Our results confirm the association between depression, depressive symptoms and sleep disorders also in persons with dementia. The high frequency of sleep disorders reported in patients with dementia could be attributable to the neuropathological mechanisms of dementia or to the co-occurrence of depression, which is the most common neuropsychiatric disorder observed in dementias $[37,38]$. However, this relationship might be bidirectional because there is also evidence that sleep disturbances can be a risk factor for depression and/or depressive symptoms [39]. No difference in the severity of cognitive impairment was observed in persons with or without any of the considered sleep disorders. This represents a controversial issue since an increase in the frequency of sleep disturbance in more severely compromised patients has been variably reported in previous studies [40-43]. Otherwise, another study which used only PSQI in a cohort of patients with com- plaints of cognitive decline, showed that poor sleep quality was more common in nondemented versus demented patients [44].

In this study, we have included community-dwelling patients with a CDR of three or lower and consequently patients with severe diseases were not represented. For this reason, an increase in frequency of sleep disorders cannot be excluded in patients with more severe symptoms than those we have studied.

$\mathrm{SDB}, \mathrm{RBD}$ and EDS were more frequent in men than in women, whereas RLS was prevalent among women and insomnia was equally distributed in the two genders. A higher prevalence in men than in women of SDB and RBD is an established knowledge about these disorders and the higher prevalence of RLS in women is in agreement with a previous study on the elderly [32].

With the exception of patients with insomnia who were significantly older, the mean ages of patients with or without any of the investigated sleep disorders were not different.

In our group of persons with cognitive impairment or dementia, 297 had one or more sleep disturbance and only $44(14.8 \%)$ of them had an isolated disturbance, showing that sleep disturbances occur almost always in association whilst a nonspecific pattern of association was found. The tendency of disorders to occur in association suggests that the neurodegenerative or vascular damage causing dementia or cognitive impairment is so diffuse in these patients that many sleep control neural circuits are affected, causing multiple different sleep disorders in the same patient.

We found that patients with $\mathrm{AD}$ and MCI had almost the same frequency of any sleep disturbance not allowing us to give them a predicting value concerning the conversion from MCI to AD. Moreover, this result was unexpected since not all the MCI patients have an incipient $\mathrm{AD}$ and almost one third are expected to return to normal after 1 or 2 years. Vascular dementia patients had a frequency of any sleep disturbance that was two times higher than that observed in AD patients. The highest risk increase with respect to $\mathrm{AD}$ was for SBD and this result is in agreement with the high frequency of obstructive sleep apneas reported in patients with stroke [15]. One likely reason for the association between vascular damage and SDB might be that the two conditions have some common risk factors such as excessive body mass index and hypertension.

Patents with FTD were two times more frequently affected than patients with $\mathrm{AD}$, although this increase was nonstatistically significant. A disruption of sleep/wake 
rhythms associated with increased nocturnal activity and decreased morning activity has already been reported in these patients [45].

LBD and PDD had the highest frequency of occurrence of any sleep disturbances that were present in $90 \%$ of the patients. In LBD and PDD patients, insomnia and clinically probable RBD were significantly more frequent than in $\mathrm{AD}$ patients. The association of REM sleep disorders and LBD has already been detected in case reports [46], and some studies have reported that RBD can precede for many years the onset of PDD and LBD [14, 47]. Our study confirms that symptoms characteristic of RBD are more frequent in LBD and PDD supporting the evidence that RBD is specifically linked to synucleinopathies [8].

In patients with LBD or PDD we did not find any subject with RLS: this is an interesting result since contradictory evidence exists about the association of Parkinson's disease and RLS $[48,49]$ and no data have been published on RLS and LBD-PDD. Our results are evidence of the absence of any clear association between RLS and these diseases characterized by movement disorders and cognitive impairment.

Some limitations of the study should be noted: we studied a community-dwelling population, spontaneously presenting to neurological centers that might be selected for specific characteristics and even for the presence and type of sleep disorders. However, the general clinical and demographic characteristics of the studied population are very similar to those of any previously reported clinical convenience sample of patients firstly diagnosed with dementia. For this reason, we believe that our findings might be extended to all the patients seen by neurologists during clinical practice. Another limit could be related to the fact that we have studied our patients only on a clinical ground not using instrumental supports. Sleep disturbances are fully investigated and diagnosed with polysomnography and/or video-polysomnography, but these techniques are complex, expensive not always well tolerated by patients and consequently difficult to obtain in a wide number of subjects. Actigraphy is also difficult to be used extensively, so instrumental supports cannot be used as a screening to detect sleep disorders.

In conclusion, our results support previous evidence that sleep disturbances are particularly frequent in persons with dementia or cognitive impairment. In cognitively impaired populations, sleep disturbances tend to occur almost invariably in association. On clinical grounds, we have been able to appreciate a higher frequency of SDB in vascular dementia and of RBD in LBD/ PDD patients. The need for accurate diagnostic criteria for defining sleep-wake disturbances in $\mathrm{AD}$ and dementias is widely emphasized [50]: an accurate clinical investigation on the presence and type of sleep disorders is relevant for a better diagnostic characterization of patients and to optimize treatments. Instrumental supports should be considered in patients with specific symptoms and signs of sleep disorders as previously detected by a careful clinical investigation.

\section{Acknowledgments}

This work was supported by a grant from the Italian Minister of Health. The authors thank the Italian National Research Center (CNR) and SIN Dem, Association for Dementia affiliated to the Italian Neurological Society (SIN), for their important contributions.

\section{References}

$>1$ Ancoli-Israel S, Ayalon L: Diagnosis and treatment of sleep disorders in older adults. Am J Geriatr Psychiatry 2006;14:95-103.

$>2$ Stepnowsky C, Ancoli-Israel S: Sleep and its disorders in seniors. Sleep Med Clin 2008;3: 281-293.

$>3$ Chokroverty S: Sleep and neurodegenerative diseases. Semin Neurol 2009;29:446-467.

$\checkmark 4$ Dauvilliers Y: Insomnia in patients with neurodegenerative conditions. Sleep Med 2007;8:27-34.

5 Vitiello MV, Borson S: Sleep disturbances in patients with Alzheimer's disease: epidemiology, pathophysiology and treatment. CNS Drugs 2001;15:777-796.

Sleep Disturbances in MCI and Dementia
6 Deschenes CL, McCurry SM: Current treatments for sleep disturbances in individuals with dementia. Curr Psychiatry Rep 2009;11: 20-26.

7 International Classification of Sleep Disorders, ed 2: Diagnostic and Coding Manual. Westchester, American Academy of Sleep Medicine, 2005.

8 Boeve BF, Silber MH, Ferman TJ, Lucas JA, Parisi JE: Association of REM sleep behavior disorder and neurodegenerative disease may reflect an underlying synucleinopathy. Movement Disord 2001;16:622-630.
-9 Harper DG, Stopa EG, McKee AC, Satlin A, Harlan PC, Goldstein R, et al: Differential circadian rhythm disturbances in men with Alzheimer's disease and frontotemporal degeneration. Arch Gen Psychiatry 2001;58: 353-360.

10 Grace JB, Walker MP, McKeith IG: A comparison of sleep profiles in patients with dementia with Lewy bodies and Alzheimer's disease. Int J Geriatr Psychiatry 2000;15:1028-1033.

11 Bracco L, Piccini C, Moretti M, Mascalchi M, Sforza A, Nacmias B, Cellini E, Bagnoli S, Sorbi S: Alzheimer's disease: role of size and location of white matter changes in determining cognitive deficits. Dement Geriatr Cogn Disord 2005;20:358-366. 
$\checkmark 12$ Peters F, Perani D, Herholz K, Holthoff V, 25 Folstein MF, Folstein SE, McHugh PR: Beuthien-Baumann B, Sorbi S, Pupi A, Degueldre C, Lemaire C, Collette F, Salmon E: Orbitofrontal dysfunction related to both apathy and disinhibition in frontotemporal dementia. Dement Geriatr Cogn Disord 2006;21:373-379.

-13 Boeve BF, Silber MH, Saper CB, Ferman TJ, Dickson DW, Parisi JE, et al: Pathophysiology of REM sleep behavior disorder and relevance to neurodegenerative disease. Brain 2007; 130:2770-2788.

- 14 Iranzo A, Molinuevo JL, Santamaría J, Serradell M, Martí MJ, Valldeoriola F, et al: Rapid-eye-movement sleep behavior disorder as an early marker for a neurodegenerative disorder: a descriptive study. Lancet Neurol 2006;5:572-577.

$\checkmark 15$ Hermann DM, Bassetti CL: Sleep-related breathing and sleep-wake disturbances in ischemic stroke. Neurology 2009;73:13131322.

16 Fuh J-L, Wang S-J, Cummings JL: Neuropsychiatric profiles in patients with Alzheimer's disease and vascular dementia. J Neurol Neurosurg Psychiatr 2005;76:1337-1341.

17 Gauthier S, Reisberg B, Zaudig M, et al: Mild cognitive impairment. Lancet 2006;367: 1262-1270.

18 Gagnon J-F, Vendette M, Postuma RB, et al: Mild cognitive impairment in rapid eye movement sleep behavior disorder and Parkinson's disease. Ann Neurol 2009;66:39-47.

19 American Psychiatric Association: DSM IVTR Diagnostic and Statistical Manual of Mental Disorders, ed 4, text revision, 2000.

-20 McKhann G, Drachman D, Folstein M, Katzman R, Price D, Stadlan EM: Clinical diagnosis of Alzheimer's disease: report of the NINCDS-ADRDA Work Group under the auspices of Department of Health and Human Services Task Force on Alzheimer's Disease. Neurology 1984;34:939-944.

-21 Román GC, Tatemichi TK, Erkinjuntti T, Cummings JL, Masdeu JC, Garcia JH, et al: Vascular dementia: diagnostic criteria for research studies. Report of the NINDS-AIREN International Workshop. Neurology 1993; 43:250-260.

22 McKeith IG: Consensus guidelines for the clinical and pathologic diagnosis of dementia with Lewy bodies (DLB): report of the Consortium on DLB International Workshop. J Alzheimers Dis 2006;9(3 suppl): 417-423.

23 Winblad B, Palmer K, Kivipelto M, Jelic V, Fratiglioni L, Wahlund L-O, et al: Mild cognitive impairment-beyond controversies, towards a consensus: report of the International Working Group on Mild Cognitive Impairment. J Intern Med 2004;256:240-246.

-24 Fillenbaum GG, Peterson B, Morris JC: Estimating the validity of the clinical Dementia Rating Scale: the CERAD experience. Consortium to Establish a Registry for Alzheimer's Disease. Aging (Milan) 1996;8:379_ 385.
'Mini-mental state'. A practical method for grading the cognitive state of patients for the clinician. J Psychiatr Res 1975;12:189-198.

26 Gallagher D, Nies G, Thompson LW: Reliability of the Beck Depression Inventory with older adults. J Consult Clin Psychol 1982;50:152-153.

27 Lawton MP, Brody EM: Assessment of older people: self-maintaining and instrumental activities of daily living. The Gerontologist 1969;9:179-186.

28 De Vreese LP, Caffarra P, Savarè R, Cerutti R, Franceschi M, Grossi E; Multicentre Study Group: Functional disability in early Alzheimer's disease - a validation study of the Italian version of the disability assessment for dementia scale. Dement Geriatr Cogn Disord 2008;25:186-194.

29 Terzano MG, Parrino L, Cirignotta F, et al: Studio Morfeo: insomnia in primary care, a survey conducted on the Italian population. Sleep Med 2004;5:67-75.

30 Netzer NC, Stoohs RA, Netzer CM, Clark K, Strohl KP: Using the Berlin Questionnaire to identify patients at risk for the sleep apnea syndrome. Ann Intern Med 1999;131:485491.

-31 Allen RP, Picchietti D, Hening WA, Trenkwalder C, Walters AS, Montplaisi J, Restless Legs Syndrome Diagnosis and Epidemiology Workshop at the National Institutes of Health, International Restless Legs Syndrome Study Group: Restless legs syndrome: diagnostic criteria, special considerations, and epidemiology. A report from the restless legs syndrome diagnosis and epidemiology workshop at the National Institutes of Health. Sleep Med 2003;4:101-119.

32 Rothdach AJ, Trenkwalder C, Haberstock J, Keil U, Berger K: Prevalence and risk factors of RLS in an elderly population: the MEMO study. Memory and morbidity in Augsburg elderly. Neurology 2000;54:1064-1068.

33 Boeve BF, Molano JR, Ferman TJ, Smith GE, Lin SC, Bieniek K, Haidar W, TippmannPeikert M, Knopman DS, Graff-Radford NR, Lucas JA, Petersen RC, Silber MH: Validation of the Mayo Sleep Questionnaire to screen for REM sleep behavior disorder in an aging and dementia cohort. Sleep Med 2011; 12:445-453.

34 Buysse DJ, Reynolds CF, Monk TH, Berman SR, Kupfer DJ: The Pittsburgh Sleep Quality Index: a new instrument for psychiatric practice and research. Psychiatry Res 1989; 28:193-213.

35 Buysse DJ, Reynolds CF 3rd, Monk TH, Hoch CC, Yeager AL, Kupfer DJ: Quantification of subjective sleep quality in healthy elderly men and women using the Pittsburgh Sleep Quality Index (PSQI). Sleep 1991; 14: 331-338. Erratum in: Sleep 1992;15:83.

36 Foley DJ, Monjan AA, Izmirlian G, Hays JC, Blazer DG: Incidence and remission of insomnia among elderly adults in a biracial cohort. Sleep 1999;22(suppl 2):S373-S378.
37 Aalten P, Verhey FR, Boziki M, et al: Neuropsychiatric syndromes in dementia. Results from the European Alzheimer Disease Consortium. Part I. Dement Geriatr Cogn Disord 2007;24:457-463.

-38 Aalten P, Verhey FR, Boziki M, et al: Consistency of neuropsychiatric syndromes across dementias: results from the European $\mathrm{Alz}$ heimer Disease Consortium. Part II. Dement Geriatr Cogn Disord 2008;25:1-8.

39 Bloom HG, Ahmed I, Alessi CA, Ancoli-Israel S, Buysse DJ, Kryger MH, Phillips BA, Thorpy MJ, Vitiello MV, Zee PC: Evidencebased recommendations for the assessment and management of sleep disorders in older persons. J Am Geriatr Soc 2009;57:761-789.

-40 Rebok GW, Rovner BW, Folstein MF: Sleep disturbance and Alzheimer's disease: relationship to behavioral problems. Aging ( $\mathrm{Mi}$ lan) 1991;3:193-196.

41 McCurry SM, Logsdon RG, Teri L, et al: Characteristics of sleep disturbance in community-dwelling Alzheimer's disease patients. J Geriatr Psychiatry Neurol 1999;12: 53-59.

42 Moran M, Lynch CA, Walsh C, Coen R, Coakley D, Lawlor BA: Sleep disturbance in mild to moderate Alzheimer's disease. Sleep Med 2005;6:347-352.

43 Prinz PN, Vitaliano PP, Vitiello MV, et al: Sleep, EEG and mental function changes in senile dementia of the Alzheimer's type. Neurobiol Aging 1982;3:361-70.

44 Hancock P, Larner AJ: Diagnostic utility of the Pittsburgh Sleep Quality Index in memory clinics. Int J Geriatr Psychiatry 2009;24: 1237-1241.

-45 Anderson KN, Hatfield C, Kipps C, Hastings M, Hodges JR: Disrupted sleep and circadian patterns in frontotemporal dementia. Eur J Neurol 2009;16:317-323.

46 Boeve BF, Dickson DW, Olson EJ, et al: Insights into REM sleep behavior disorder pathophysiology in brainstem-predominant Lewy body disease. Sleep Medicine 2007;8: 60-64.

47 Britton TC, Chaudhuri KR: REM sleep behavior disorder and the risk of developing Parkinson disease or dementia. Neurology 2009;72:1294-1295.

48 Calzetti S, Negrotti A, Bonavina G, Angelini $\mathrm{M}$, Marchesi E: Absence of co-morbidity of Parkinson's disease and restless legs syndrome: a case-control study in patients attending a movement disorders clinic. Neurol Sci 2009;30:119-122.

-49 Lee JE, Shin H-W, Kim KS, Sohn YH: Factors contributing to the development of restless legs syndrome in patients with Parkinson disease. Movement Disord 2009;24:579-582.

50 Yesavage JA, Friedman L, Ancoli-Israel S, Bliwise D, Singer C, Vitiello MV, Monjan AA, Lebowitz B: Development of diagnostic criteria for defining sleep disturbance in Alzheimer's disease. J Geriatr Psychiatry Neurol 2003;16:131-139. 